\title{
Microbiology and management of brain abscess in children
}

\author{
Itzhak Brook
}

Department of Pediatrics Georgetown University School of Medicine Washington, DC, U.S.A.

\begin{abstract}
Brain abscess is an uncommon but serious life threatening infection in children. The infection can originate from contiguous sites of existing infections, such as chronic otitis media, dental infection, mastoiditis, or sinusitis. The infection can also occur in children with cyanotic congenital heart disease where the predominant organisms are viridans, microaerophilic, or anaerobic streptococci or following head trauma where Staphylococcus aureus, viridans Streptococci and Streptococcus pneumoniae are the most prevalent isolates. Brain scans, computerized tomography and magnetic resonance imaging are important tools that enable accurate diagnosis of the infection. Proper selection of antimicrobial with good intracranial penetration is critical in the management of intracranial infection. Brain abscess, especially in the early phase of cerebritis, may respond to antimicrobial therapy without the need for surgical drainage. Delay in surgical drainage can be associated with high mortality or morbidity. (J Pediatr Neurol 2004; 2(3): 125-130).
\end{abstract}

Key words: brain abscess, subdural empyema, sinusitis, otitis media, Staphylococcus aureus.

\section{Introduction}

Brain abscess is an uncommon but serious life threatening infection in children. It can be take the form of a brain abscess or subdural or

Correspondence: Itzhak Brook, M.D., MSc

4431 Albemarle st NW

Washington DC 20016 U.S.A.

Tel: 301295 2698, fax: 2530818709 .

E-mail: ib6@georgetown.edu

Received: January 07, 2004.

Revised: February 09, 2004.

Accepted: March 07, 2004. extradural empyema, and be classified according to its anatomic location or microbial etiology. Brain abscess can result from infection of contiguous structures (i.e. dental infection, otitis media, mastoiditis, and sinusitis), as the consequence of hematogenous spread from a remote location (lung infections, bronchiectasis in those with cystic fibrosis, esophageal dilatation, endoscopy, pulmonary arteriovenous malformations), especially in those with cyanotic congenital heart disease; after penetrating head trauma or surgery; or following meningitis. In some patients no clear source can be identified.

This review summarizes the studies of the microbiology and the recent developments in the diagnosis and management of brain abscess in children.

\section{Pathophysiology}

The infection can enter the intracranial compartment by several possible routes (1) direct extension through necrotic areas of osteomyelitis such as the posterior wall of the frontal sinus. This direct route of intracranial extension is more commonly associated with chronic ear infection than with sinusitis (2). Contiguous spread could extend to various sites in the central nervous system (CNS), causing cavernous sinus thrombosis, retrograde meningitis, and epidural, subdural, and brain abscess. The valveless venous network that interconnects the intracranial venous system and the vasculature of the sinus mucosa provides an alternative route of intracranial bacterial entry. Thrombophlebitis originating in the mucosal veins progressively involves the emissary veins of the skull, the dural venous sinuses, the subdural veins, and finally the cerebral veins. By this route of spread, the subdural space may be selectively infected without contamination of the intermediary structure; that is, a subdural empyema can exist without extradural infection or osteomyelitis. Intracranial extension of the infection by the venous mode is common in paranasal sinus disease, especially in acute exacerbation of chronic 
Table 1. Predominant organisms causing brain abscesses in children

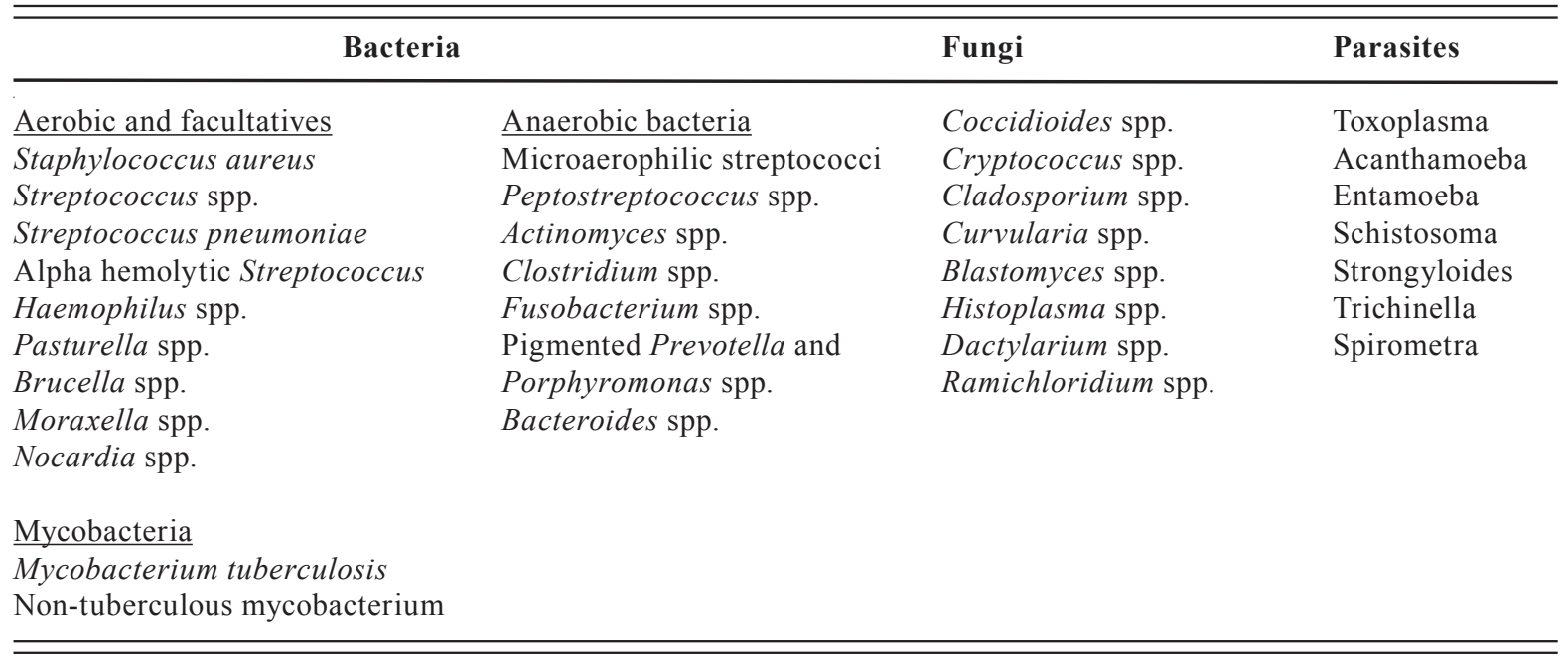

inflammation. (3). Trauma that can cause open fracture or following neurosurgery can allow direct seeding of organisms to the brain (4). Hematogenous spread from a distant focus is a common cause. The source of the infection is unknown in up to about one fifth of patients. Intracranial complications can also develop secondary to periapical abscess of the upper incisors through hematogenous spread or through secondary maxillary sinusitis (3).

\section{Clinical presentation}

The clinical course varies from indolent to fulminant. In about $2 / 3$ rds of patients, the symptoms last for up to 2 weeks before diagnosis is made. The infection occurs more frequently in males. Because the main predisposing cause of subdural empyema in young children is bacterial meningitis, the decrease in meningitis due to Haemophillus influenzae vaccination has reduced the incidence of this infection in children. A sudden deterioration in the child clinical condition, often suggests a rupture of a brain abscess into the ventricles or subarachnoid space.

The clinical presentation of brain abscess is usually manifested by symptoms of a spaceoccupying lesion. The symptoms and signs include: fever, persistent headache that often is localized, drowsiness, confusion, stupor, general or focal seizures, nausea and vomiting, focal motor or sensory impairments, papilledema, ataxia, hemiparesis. Mental changes, or general seizures are observed in less than half of the patients. Localized neurological signs are eventually found in most patients. In the initial stages of the infection, a brain abscess can present as encephalitis accompanied by signs of increased intracranial pressure. Papilledema in the older child or bulging fontanels may be present in about a third of younger infant. A ruptured brain abscess can produce purulent meningitis.
Often, a frontal lobe and parietal lobe abscesses are not symptomatic for a long time, until a mass effect is achieved.

\section{Microbiology}

Table 1 shows the predominant organisms causing brain abscesses in children. Anaerobic and microaerophilic cocci and Gram-negative and Grampositive anaerobic bacilli are the most important isolates. These include Bacteoides spp., Prevotella spp., Fusobacterium spp., and Clostridium spp. (411).

The predominant aerobic and facultative organisms were Staphylococcus aureus, Streptococcus pneumoniae, Streptococcus pyogens, and alpha-hemolytic streptococci. Less common causes include Escherichia coli, Proteus spp., Citrobacter spp., Klebsiella spp., Salmonella spp., Enterobacter spp., Psuedomonas spp., Haemophilus influenzae, Haemophilus aphrophilus, Moraxella spp., Eikenella spp., Brucella spp., Bacillus cereus, Streptococcus pneumoniae, Neisseria meningitidis and Nocardia asteroids. Other agents include mycobacteria, fungi, and parasites ( i.e. Entamoeba histolytica, Toxoplasma, Acanthamoeba, Paragonimus, Schistosoma, Spirometra, and Trichinella) (12).

Differential diagnosis includes infectious and non-infectious etiologies. The infectious include: encephalitis, meningitis, mycotic aneurism, cranial osteomyelitis, epidural abscess, subdural empyema, supputative thrombophlebitis, cysticercosis, cryptococcosis. The non-infectious etiologies include: hemorrhage, venous sinus thrombosis, cerebral infarct, migraine, primary or metastatic tumor.

\section{Diagnosis}

Routine tests include: complete blood count, 
serum C-Reactive protein, blood cultures. There is generally moderate leukocytosis and erythrocyte sedimentation rate and C-Reactive protein are generally elevated. However, in less than a third of the patients with leukoytosis the number of leukocytes is less than $20,000 / \mathrm{mm}^{3}$ Serum sodium levels can be lowered as a result of inappropriate anti-diuretic hormone production. Lumbar puncture should not be done until increased intracranial pressure has been excluded by computed tomography $(\mathrm{CT})$ or magnetic resonance imaging (MRI). Hypoglycorrhachia $(<40$ $\mathrm{mg} / \mathrm{dL}$ ) is seen only in a third of the patients, and elevated protein $(>40 \mathrm{mg} / \mathrm{dL})$ is present in two third. The usual CSF findings associated with subdural or parencymal abscesses consist of an elevated protein, pleocytosis with a variable neutrophil count, a normal glucose, and sterile cultures.

The number of leukocytes is often elevated and reached $100,000 / \mathrm{mm}^{3}$ or more when a rupture of the abscess occurs into the CSF. Numerous red blood cells generally are observed at that time, and the CSF lactic acid is then elevated above 500 $\mathrm{mg} \%$. CSF cultures are positive only in less than $10 \%$ of patients, unless the abscess has ruptured or a concomitant meningitis exists. Aspirates of abscesses should be cultured for aerobic, anaerobic, fungi, and acid-fast organisms and appropriate staining should be performed.

\section{Radiological and other studies}

The preferred initial method of evaluating a child suspected of a brain abscess is MRI with and without gadolinium enhancement. Identical results can be obtained from cranial CT scans with or without the intravenous administration of iodinated contrast material. Both imaging techniques assist in detecting the mass effect of the abscess; however, findings in MRI with a diffusion protocol are more specific in differentiating between cerebral tumor, stroke, and abscess.

Evaluating the metabolite peaks with magnetic resonance spectroscopy can differentiate tumor, from radiation necrosis, and abscess. Perfusion MRI can be used to differentiate these lesions by evaluating vascularity with blood flow analysis with dynamic intravenous gadolinium contrast injection studies.

Distinguishing brain abscess from neoplasm or postoperative changes from infection is difficult on occassion. In these individuals, a nuclear marker can be utilized to tag white blood cells or antibodies to help differentiation.

Plain radiographs of the paranasal sinuses can only suggest a potential etiology for cerebral abscess. Early findings of CT examinations are not specific for brain abscess. In some children the edema pattern and moderate mass effect cannot be differentiated from tumor or stroke. MRI results in patients with cerebritis may mimic findings in stroke, while findings in the infarcts resulting from vasculitis and cerebritis may be similar to those of embolic strokes. Nuclear medicine single photon emission computed tomographic studies are not specific for brain abscess unless a white cell tag is used.

Imaging techniques are helpful in detecting the size, number, and location of an abscess and has become the mainstay of diagnosis and follow-up. The typical findings of a CT include a hypodense center, surrounded by ring enhancement, and another area of hypodensity, which is due to the edema of the tissues surrounding the abscess. (Figure 1). It provides a rapid means of confirming the diagnosis, localizing the lesion, and monitoring the progression of the abscess after treatment has been initiated. However CT can lag behind clinical finding by a few days. Since the advent of CT and MRI scanning, the case fatality rate has fallen by $90 \%$. Arteriograms and ventriculograms are invasive techniques that are infrequently indicated (13).

MRI is considered the diagnostic method of choice. It permits accurate diagnosis and excellent follow-up of the lesions because of its superior sensitivity and specificity as compared to CT. Electroencephalogram occasionally can discover a focus of high voltage with slow activity; however, this is the least accurate procedure in the diagnostic evaluation.

High resolution ultrasonography may can assist in detection of subdural empyema in infants, and it's differentiation from subdural effusion. CT can also recognize subdural empyema. The preferred method is the contrast enhanced CT, which delineating the inflammatory response of the cerebral cortex or the meninges.

\section{Treatment}

Before the abscess has become encapsulated and localized, antimicrobial therapy accompanied by measures to control the increase in the intracranial pressure are essential. Once an abscess has formed, surgical excision or drainage combined with a long a course of antibiotics ( 4 to 8 weeks) remains the treatment of choice. Some neurosurgeons advocate complete evacuation of the abscess, while others advocate repeated aspirations as indicated. Success in treating of brain abscess with antibiotic alone have been well documented (14). For this approach to be successful the patient need to be stable without increases intracranial pressure, have symptoms for less than 2 weeks, and have an abscess that is less than $2 \mathrm{~cm}$ in diameter.

In cases with multiple abscesses, those with 


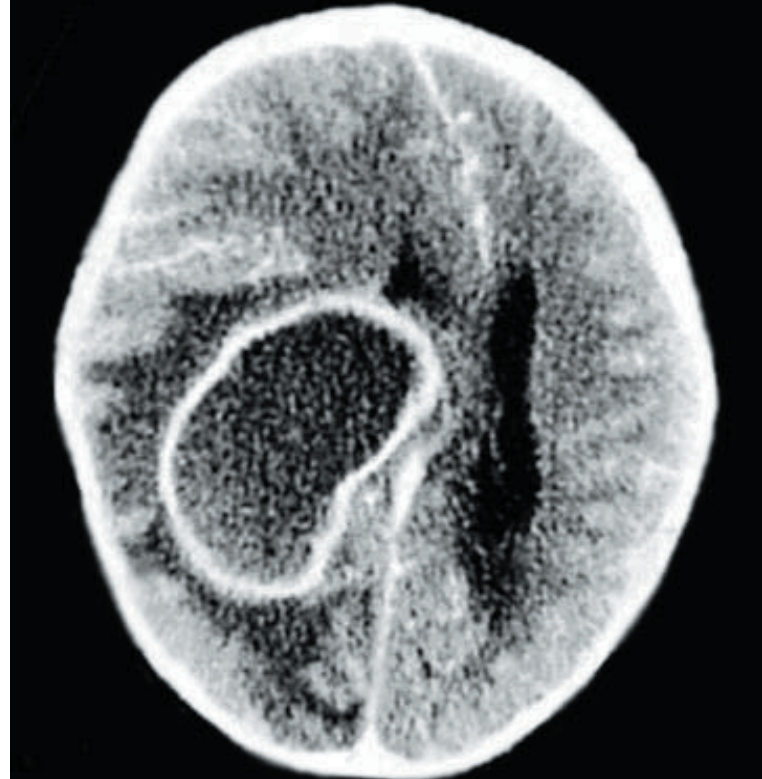

Figure 1. A 2-year-old child with abrain abscess. Head CT shows capsule which enhances with IV contrast and cerebral edema and midline shift (Courtesy of Dr. John J. Mickell M.D., Children's Medical Center of the Medical College of Virginia U.S.A.).

abscesses in essential brain areas, those with concomitant meningitis of unkown etiology, and those who are poor surgical risk, repeated aspirations are preferred to complete excision. High-dose antibiotics for an extended period may represent an alternative approach in this group of patients.

The use of CT or MRI guided stereotactic techniques facilitated the diagnosis and management of brain abscesses. An early effort at making a microbiologic diagnosis is important in planning appropriate antimicrobial therapy. The introduction of CT-guided needle aspiration can provide this important information (15). The patient must be followed carefully with frequent imaging studies. A repeat imaging study is indicated at least once a week in the acute stage. An earlier CT scan is indicated only if there is no clinical response. Although surgical intervention remains an essential treatment, selected patients may respond to antibiotic alone.

The corticosteroids use for treatment of brain abscess is controversial (16). Steroids can retard the encapsulation process, increase necrosis, reduce antibiotic penetration into the abscess and alter CT scan images. Steroid therapy can also produce a rebound effect when discontinued. When they are used to reduce cerebral edema, therapy should be for a short duration. The appropriate dosage, the proper timing and the effect of steroid therapy on the course of the disease are unknown. However, steroides use can be life saving and they should be given to those with increased intracranial pressure and/or neurological deterioration.

A number of factors should be considered when trying to decide the appropriate approach to therapy. Abscesses with a mean size of $1.7 \mathrm{~cm}$ (range 0.8 to $2.5 \mathrm{~cm}$ ) generally responded to antimicrobial therapy, while those who failed had an average pretreatment size of $4.2 \mathrm{~cm}$ (range 2.0 to $6.0 \mathrm{~cm}$ ) (17).

Knowledge of the etiological agent(s) can allow for appropriate selection of antimicrobial(s). Duration of the symptoms before diagnosis is an important factor. Bacterial abscess in the brain is preceded by infarction and cerebritis. Initiating antibiotic therapy during the early stage when there is no evidence of expanding mass lesion can prevent the progress from cerebritis to abscess. Furthermore, patients who have symptoms for less than one week have a more favorable response to medical therapy than those patients with symptoms greater than one week. Patients treated with medical therapy alone usually demonstrate clinical improvement before significant changes in the CT scan can be seen. The CT and MRI scans should eventually show a decrease in size of the lesion, a decrease in accompanying edema, and lessening of the enhancement ring. Improvement on CT scan is generally observed within one week to four weeks (average 2.5 weeks), and complete resolution is one month to 11 months (average 3.5 months).

The antimicrobial therapy of the brain abscess is generally long ( 4 to 8 weeks) because of the prolonged time needed for brain tissue to repair and close abscess space. However, a shorter course can be given if surgical drainage is achieved. Because of the difficulty involved in the penetration of various antimicrobial agents through the blood-brain barrier, the choice of antibiotics is restricted (18).

Penicillin penetrates well into the abscess cavity and is active against non-beta lactamase producing anaerobes and aerobic organisms. Chloramphenicol penetrates well into the intracranial space, and is active also against Haemophilus spp., S. pneumoniae and most obligate anaerobes. Metronidazole penetrates well into the CNS, but is only active against strict anaerobic bacteria. Third-generation cephalosporins (i.e., cefotaxime, ceftazidime) are generally adequate therapy of aerobic Gram-negative organisms. Aminoglycosides do not penetrate well into the CNS, and are relatively less active because of the anaerobic conditions and the acidic contents of the abscess. Beta lactamase resistant penicillins (i.e., oxacillin, methicillin, and nafcillin) provide good coverage against $S$. aureus. However, their penetration into the CNS is less than penicillin. Vancomycin is the most effective against meticillinresistant $S$. aureus and $S$. epidermidis as well as aerobic and anaerobic streptococci and Clostridium spp. 
Table 2. The choice of combinations of empiric therapy according to the predisposing factors

1. For meningitis: in neonates: cefotaxime+ampicillin; in infants and children: cefotaxime or ceftriaxone+ vancomycin.

2. For cyanotic congenital heart disease: ceftriaxone+metronidazole, or ampicillin + chloramphenicol, or ampicillin-sulbactam.

3. For otitis or sinusitis: Ceftriaxone+metronidazole, or ampicillin+chloramphenicol, or ampicillin-sulbactam.

4. For VP shunt or trauma: Vancomycin+anti Pseudomonal cephalosporin (i.e. ceftazidime, cefepime).

5. For the immunocompromised: vancomycin + ceftazidime + metronidazole. Amphotericin B should be added if no response takes place within 7 days.

6. For endocarditis: in natural valve: ampicillin or penicillin +cefriaxone or an aminoglycoside; in prostatic valve: vancomycin+gentamicin or ceftazidime.

With the exception of $B$. fragilis group and some strains of Prevotella spp., Porphyromonas spp. and Fusobacterium spp. most of the anaerobic pathogens isolated are sensitive to penicillin (19). However, since these penicillin-resistant anaerobic organisms predominate in brain abscess, empiric therapy should include agents effective against them that can also penetrate the blood-brain barrier. These include metronidazole, chloramphenicol, ticarcillin, plus clavulanic acid or a carbapenem (i.e. imipenem, meropenem). Caution should be used in administering imipenem, as high doses of this agent were associated with seizure activity. Penicillin should be added to metronidazole to cover aerobic and microaerophilic streptococci. The administration of a beta-lactamase-resistant penicillin or vancomycin for the treatment of $S$. aureus is generally recommended. Initial empiric antimicrobial therapy should be based on the expected etiological agents according to the likely primary infection source. When organisms are isolated, the initial empiric therapy can be adjusted to treat the specific bacteria.

The choice of combinations of empiric therapy according to the predisposing factors are include in Table 2. Injection of antibiotics into the abscess cavity was advocated in the past in an effort to sterilize the area before operation; however, because many antimicrobials penetrate brain abscess cavities fairly well, installation of antibiotics into the abscess after drainage is not needed.

Nocardia infection is generally treated with trimethoprim-sulfamethoxazole, and fungal infections are treated with amphotericin B, with or without flucytosine. If not recognized early, both subdural empyema and brain abscess can be fatal although antibiotics have improved the outlook for patients with these diseases. Management of subdural empyema requires prompt surgical evacuation of the infected site and antimicrobial therapy. Failure to perform surgical drainage can lead to a higher mortality rate.

Although proper selection of antimicrobial therapy is of primary importance in the management of intracranial infections, surgical drainage may be required (20). Delay in surgical drainage and decompression can be associated with high morbidity and mortality; however, recent studies illustrated that brain abscess in the early phase of cerebritis may respond to antimicrobial therapy without surgical drainage; however, surgical drainage may be necessary in many patients to ensure adequate therapy and complete resolution of infection (21).

Patients who do not meet the criteria for medical therapy alone, require surgery. Two surgical approaches are available: stereotactic aspiration, and excision. The risk of repeated aspiration is that the procedure may causes bleeding. Excision is especially indicated in posterior fossa or multiloculated abscess, and those that are caused by fungi or helminths, and those that re-accumulate following repeated aspirations.

\section{Complication}

Mortality has been reduced since the introduction of effective antimicrobial and the availability of CT and MRI. Mortality currently is less than 5-15\% (22). The prognosis of uncomplicated subdural abscess is very good when therapy is instituted before the emergence of radicular symptoms. The long term outcome depends on the length of symptoms prior to surgery and the degree of compression.

\section{References}

1. Grigoriadis E, Gold WL. Pyogenic brain abscess caused by Streptococcus pneumoniae: case report and review. Clin Infect Dis 1997; 25: 1108-1112.

2. Rosenfeld EA, Rowley AH. Infectious intracranial complications of sinusitis, other than meningitis, in children: 12-year review. Clin Infect Dis 1994; 18: 750-754.

3. Brook I, Friedman EM, Rodriguez WJ, Controni G. Complications of sinusitis in children. Pediatrics 1980; 66: 568-572.

4. Idriss ZH, Gutman LT, Kronfol NM. Brain abscesses in infants and children: current status of clinical findings, management and prognosis. Clin Pediatr 1978; 17: 738-740, 745-746.

5. Fischer EG, McLennan JE, Suzuki Y. Cerebral 
abscess in children. Am J Dis Child 1981; 135: 746 749 .

6. Ayyagari A, Pamcholi VK, Kak VK, et al. Bacteriological spectrum of brain abscess with special reference to anaerobic bacteria. Indian J Med Res 1983; 77: 182-186.

7. Jadavji T, Humphreys RP, Prober CG. Brain abscesses in infants and children. Pediatr Infect Dis 1985; 4: 394-398.

8. Chun CH, Johnson JD, Hofstetter M, Raff M. Brain abscess. A study of 45 consecutive cases. Medicine 1986; 65: 415-431.

9. Aebi C, Kaufmann F, Schaad UB. Brain abscess in childhood--long-term experiences. Eur J Pediatr 1991; 150: 282-286.

10. Saez-Llorens XJ, Umana MA, Odio CM, McCracken GH Jr, Nelson JD. Brain abscess in infants and children. Pediatr Infect Dis J 1989; 8: 449-458.

11. Brook I. Aerobic and anaerobic bacteriology of intracranial abscesses. Pediatr Neurol 1992; 8: 210214.

12. Yogev R. Focal suppurative infections of the central nervous system. In: Long SS, Pickering LK, Prober CG (eds). Principles and Practice of Pediatric Infectious Diseases (2nd ed). New York: Churchill Livingstone, 2003, pp 302-312.

13. Nathoo N, Nadvi SS, van Dellen JR, Gouws E. Intracranial subdural empyemas in the era of computed tomography: a review of 699 cases.
Neurosurgery $1999 ; \mathbf{4 4 :}$ 529-535.

14. Carpenter JL. Brain stem abscesses: cure with medical therapy, case report, and review. Clin Infect Dis 1994; 18: 219-226.

15. Fuentes S, Bouillot $P$, Regis J, Lena G, Choux M. Management of brain stem abscess. Br J Neurosurg 2001; 15: 57-62

16. Han YY, Sun WZ. An evidence-based review on the use of corticosteroids in peri-operative and critical care. Acta Anaesthesiol Sin 2002; 40: 71-79.

17. Boom WH, Tuazon CU. Successful treatment of multiple brain abscesses with antibiotics alone. Rev Infect Dis 1985; 7: 189-199.

18. Everett ED, Strausbaugh LJ. Antimicrobial agents and the central nervous system. Neurosurgery 1980; 6: $691-714$

19. Hecht DW. Evolution of anaerobe susceptibility testing in the United States. Clin Infect Dis 2002; 35 Suppl 1: 28-35.

20. Ciurea AV, Stoica F, Vasilescu G, Nuteanu L. Neurosurgical management of brain abscesses in children. Child"s Nerv Syst 1999; 15: 309-317.

21. Donaldson G, Webster D, Crandon IW. Brain abscess at the University Hospital of the West Indies. West Indian Med J 2000; 49: 212-215.

22. Tekkök IH, Erbengi A. Management of brain abscess in children: review of 130 cases over a period of 21 years. Child's Nerv Syst 1992; 8: 411-416. 